\title{
Séchage du sorgho produit dans le Nord-Bénin : Caractérisation thermophysique et qualité microbiologique du produit séché
}

\author{
Pie Savin GANDAHO1, Codjo GOUDJINOU1, Fidèle P. TCHOBO1, Mohamed M. SOUMANOU1* \\ 1 Unité de Recherche en Génie Enzymatique et Alimentaire (URGEA), Laboratoire d'Étude et de Recherche en Chimie \\ Appliquée (LERCA), Département de Génie de Technologie Alimentaire, École Polytechnique d'Abomey-Calavi (EPAC), \\ Université d'Abomey-Calavi (UAC), 01 BP 2009 Cotonou, Bénin. \\ *Auteur correspondant : ocdacm@gmail.com Tél : (+229) 97726460
}

Original submitted in on 25 December 2016. Published online at www.m.elewa.org on $30^{\text {th }}$ April 2017 https://dx.doi.org/10.4314/jab.v112i1.6

\section{RESUME}

Objectif : Le présent travail a consisté à évaluer l'effet de deux (02) méthodes de séchage sur les propriétés cinétiques et la qualité microbiologique du sorgho produit au Nord-Bénin.

Méthodologie et résultats : Des échantillons de sorgho de variété « tokobèssèmi » sont séchés sous abri et en plein air pendant (30) jours dans cinq (05) communes du département de l'Alibori. Les paramètres thermiques tels que la cinétique de séchage, l'influence de l'humidité relative et de la température de l'air asséchant sur la teneur en eau du sorgho ont été déterminés. Une analyse microbiologique des échantillons de sorgho séchés a permis la détermination de la flore totale, des levures et moisissures, des coliformes totaux, des coliformes fécaux et/ou thermo tolérants et des Staphylococcus aureus. Les résultats ont montré que l'augmentation de la température de séchage et la diminution de l'humidité relative de l'air asséchant permettent d'obtenir des durées de séchage courtes et de faibles teneurs en eau du produit. La flore totale des échantillons de sorgho est remarquable de même que les coliformes totaux et fécaux, levures et moisissures sont également présents. Les microorganismes indicateurs de qualité tels que, Staphylococcus aureus sont absents. Les taux de germes fécaux varient entre 300 à 43000 UFC /g en plein air et 84 à 37000 UFC /g sous abri. Les coliformes totaux peu nombreux à Kandi sont absents dans les autres échantillons en plein air. Ils sont quasiment absents sous abri. En plein air les levures varient entre 23 (Gogounou) et 92 UFC/g (Malanville) pendant que la densité de moisissures quoi que faible reste à 41 à Gogounou. Sous abri ces germes ne sont pratiquement pas observés. Conclusion et application des résultats : Le séchage sous abri en milieu paysan présente apparemment un caractère sanitaire acceptable pour l'alimentation humaine au vu des critères de qualité tels que définis par les micro-organismes indicateurs.

Mots clés : Séchage, Sorgho, Comportement cinétique, Qualité microbiologique, Bénin. 
Sorghum drying produced in North Benin: Thermophysical characterization and microbiological quality of the dried product

\section{ABSTRACT}

Objective: This work evaluates the influence of two (02) drying methods on the kinetic properties and microbiological quality of sorghum produced in North Benin.

Methodology and results: Sorghum samples of the variety "tokobèssèmi" were dried under shelter and outdoors for (30) days in five (05) villages in the Department of Alibori. Thermical parameters such as the drying kinetics, the influence of relative humidity and air temperature drying on sorghum water content were determined. Microbiological analysis of samples of dried sorghum is paid to the determination of the total flora, yeast and mold, total coliform, fecal coliform and / or thermo tolerant and Staphylococcus aureus. The results showed that the increase temperature and a relative decrease in moisture from the drying air possible to obtain short drying times and low water contents of the product. The total flora of the sorghum samples is remarkable as well as total and faecal coliforms, yeasts and molds are also present. Microorganism's quality indicators such as Staphylococcus aureus were absent. Rates vary between faecal microbes 300-43 000 outdoor and 84-37000 indoors. The small total coliforms in Kandi are absent in the samples of other outdoor departments. They are almost absent under cover. Outdoor yeast number varies between 23 (Gogounou) and 92 (Malanville) while the mold of whatever density remains low at 41 to Gogounou. Under shelter these microbes are practically not observed.

Conclusion and application of findings: Drying under a shelter in a farming environment apparently has an acceptable sanitary character for human food in view of the quality criteria as defined by the indicator microorganism.

Keywords: Drying, Sorghum, Kinetic behavior, Microbiological quality, Benin.

\section{INTRODUCTION}

La conservation des denrées alimentaires pendant la phase post-récolte constitue une préoccupation majeure pour les producteurs dans l'espace rural. En effet, la plus grande partie de notre alimentation est composée de produits agricoles qui sont généralement saisonniers et hautement périssables (Touz et Merzaia-Blama, 2008). Pour disposer donc d'aliments tout au long de l'année, certaines méthodes traditionnelles permettant de prolonger la durée du stockage des produits, ont été mises au point. Ces technologies traditionnelles généralement mises en œuvre pour la conservation des denrées alimentaires, sont souvent inadéquates avec des risques d'infestation des produits stockés par des moisissures toxinogènes et autres (Fandohan et al., 2003). Ainsi, $25 \%$ des récoltes dans le monde sont contaminées par les mycotoxines provenant des moisissures toxinogènes, ce qui a pour conséquence la réduction de la quantité d'aliments disponibles au plan mondial (Yiannikouris et Jouany, 2002). Au nombre des techniques de conservation traditionnellement connues, le séchage à l'air libre occupe une place de choix dans les pays en développement. En effet, ce type de séchage expose les produits aux aléas et aux risques de contamination par la flore aéroportée. Le séchage naturel des produits agricoles est un mode traditionnel de séchage solaire qui consiste à utiliser l'action du rayonnement solaire et des mouvements convectifs de l'air ambiant pour réduire la teneur en eau afin de mieux conserver le produit (Benhamou et al., 2008). Souvent, les séchoirs mis au point pour remédier aux imperfections de la méthode traditionnelle basée sur le séchage solaire ne sont pas toujours en adéquation avec les réalités socioéconomiques du monde rural (Kouhila, 2001). Le séchage maîtrisé et amélioré peut contribuer considérablement au renforcement de la sécurité alimentaire et sanitaire des produits. II est alors nécessaire d'examiner toutes les possibilités d'élaboration et d'utilisation de méthodes de conservation de performances relativement élevées et compatibles avec la situation socio-économique et les conditions climatiques du milieu. Le présent travail vise à étudier l'influence de deux modes de séchage (plein air et sous abri) sur la teneur en eau 
et la qualité microbiologique du sorgho produit dans cinq (05) communes du Nord-Bénin.

\section{MATERIEL ET METHODES}

Matériel végétal : Le sorgho blanc de Bagou est récolté dans les champs dans cinq (05) communes de l'Alibori (Malanville, Gogounou, Ségbana, Banikoara et Kandi). II a été d'abord stocké au champ dans les greniers et est transporté par la suite au lieu de séchage. La figure1 qui suit, présente la carte de situation géographique des localités d'étude.

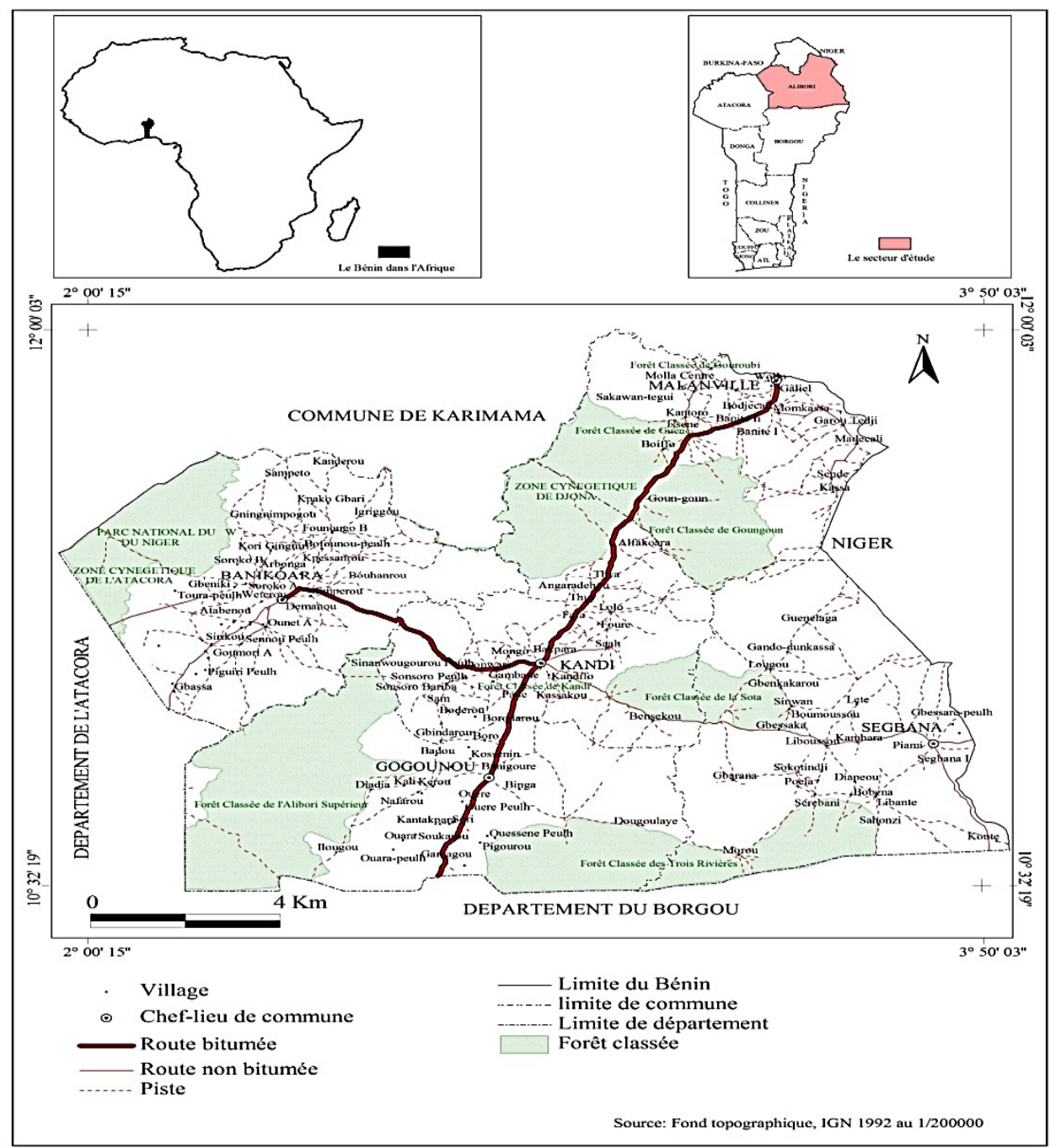

Figure 1 : Carte montrant la situation géographique de la zone d'étude.

Préparation de la matière première: Le sorgho en panicule est battu pour libérer les grains destinés au séchage. Deux méthodes de séchage (plein air et sous abri) ont été utilisées dans cette étude. Le séchage en plein air des grains de sorgho est fait au sol par exposition directe au soleil. Les grains de sorgho sont pesés et répartis en couche mince sur des sachets plastiques transparents de $(2 \mathrm{~m} \times 1,5 \mathrm{~m})$. La densité de chargement de chaque sachet est $3 \mathrm{~kg} / \mathrm{m}^{2}$ de produit frais. Les échantillons sont uniformément et régulièrement répartis en couche mince afin d'assurer une homogénéité de diffusion lors de l'opération de séchage (Figure 2). Le séchage sous abri est réalisé dans une pièce circulaire de $3 \mathrm{~m}$ de diamètre et de $3,5 \mathrm{~m}$ de hauteur construit à cet 
effet. Cette pièce est munie de palier translucide de $2 \mathrm{mx}$ $90 \mathrm{~cm}$ laissant passer facilement la lumière du soleil. Les faces latérales de la pièce sont grillagées pour la diffusion simple de l'air et dans le même temps empêcher l'intrusion des ravageurs. Dans ces conditions, les grains de sorgho sont pesés et répartis en couche mince sur des

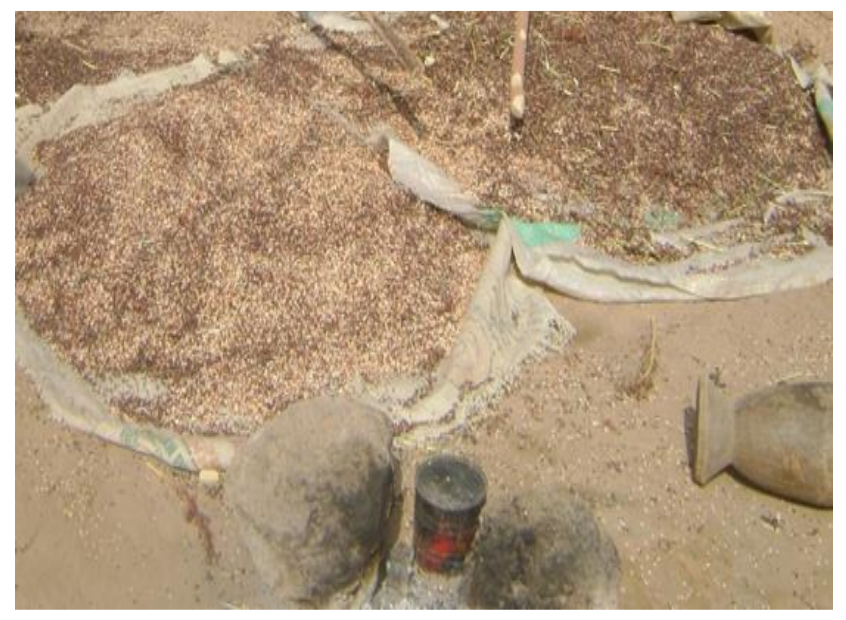

Figure 2 : Séchage de sorgho en plein air

Détermination de la température et de l'humidité relative de l'air asséchant: La température de l'ambiance de séchage et l'humidité relative de l'air asséchant sont régulièrement mesurées tous les cinq (05) jours à l'aide du data logger. Le data logger EL-USB-2 RH- TEMP DATA LOGGER. L'appareil est programmé pour des fréquences de temps bien déterminées.

Détermination de la teneur en eau des échantillons du sorgho: La mesure en continue de la perte de masse des échantillons du sorgho au cours du temps est effectuée à l'aide d'une balance toutes les semaines pendant un mois. A la fin de chaque expérience, les échantillons sont repris puis immédiatement placés dans l'étuve de dessiccation réglée à $103^{\circ} \mathrm{C} \pm 2$. Cette opération permet d'obtenir les extraits secs des échantillons étudiés, qui subissent dans cette condition la perte totale des eaux libres et liées qu'ils contiennent (Ahouannou et al., 2010). Les échantillons sont régulièrement pesés et si au bout de 24 heures leurs masses ne varient plus $(0,001 \mathrm{~g})$ alors l'extrait sec est obtenu. Ainsi, la masse mesurée pour chaque échantillon est considérée comme la masse anhydre (Ms). A partir de ce paramètre obtenu, nous avons défini les différentes teneurs en eau à l'équilibre des échantillons. La teneur en eau à l'équilibre du produit sur base sèche est calculée par l'expression suivante : tôles transparentes perforées peintes en noir mat $(2 \mathrm{~m} \mathrm{x}$ $0,9 \mathrm{~m})$. La densité de chargement de chaque sachet est $3 \mathrm{~kg} / \mathrm{m}^{2}$ de produit frais. La température moyenne de la chambre est de $30 \pm 1^{\circ} \mathrm{C}$. Le séchage est contrôlé par convection naturelle (Figure 3 ).

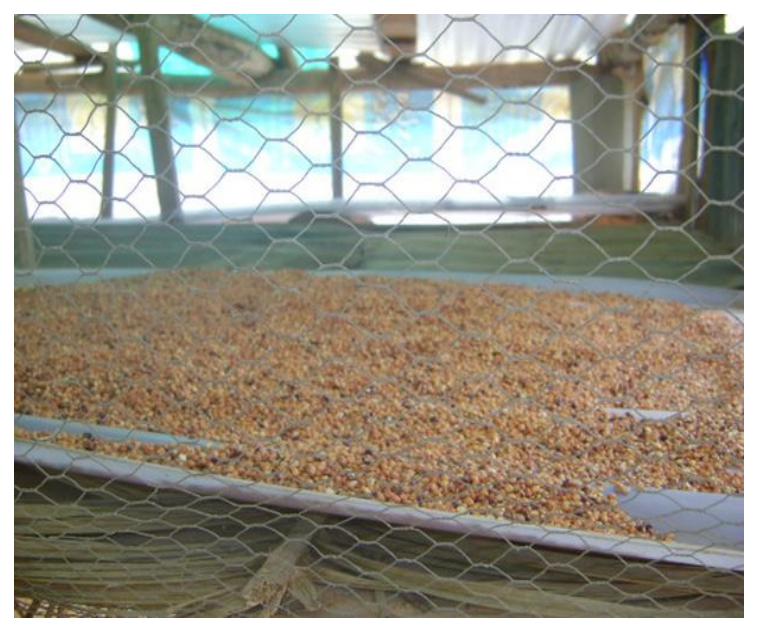

Figure 3 : Séchage de sorgho sous abri

$X_{\varepsilon q}(\%)=100 \times \frac{\left(M_{z q}+M_{s}\right)}{M_{s}}$

Xeq : Teneur en eau à l'équilibre de l'échantillon, en \% sur base sèche ; Me : Masse à l'équilibre de l'échantillon testé, en $\mathrm{kg}$; Ms : Masse anhydre de l'échantillon testé, en $\mathrm{kg}$.

Caractérisation microbiologique des échantillons de sorgho : Les analyses microbiologiques ont été réalisées suivant la méthode décrite par Guiraud et Galzy (1980). Les micro-organismes dénombrés dans les échantillons de sorgho séchés suivant les deux méthodes sont la flore totale, les levures et moisissures, les coliformes totaux, fécaux et $/$ ou thermotolérants et les germes Staphylococcus aureus. Toutes les déterminations ont été effectuées en trois exemplaires.

Analyses statistiques: Les analyses statistiques des données ont été effectuées à l'aide des logiciels STATISTICA (Program for Social Sciences, version 11). Les comparaisons entre les variables dépendantes ont été déterminées au moyen de l'analyse de variance (ANOVA) à deux facteurs. La signification statistique a été définie à $p \leq 0,05$. 


\section{RESULTATS ET DISCUSSIONS}

Courbes cinétiques de séchage du sorgho: Les courbes cinétiques de séchage du sorgho en plein air et sous abri des différents sites d'expérimentation sont respectivement présentées par les figures 4 et 5 . Ces courbes expriment l'évolution de la teneur en eau réduite en fonction du temps de séchage. Les différentes courbes de séchage obtenues montrent une allure décroissante. Cependant, on note l'absence de la phase 0 (phase à allure croissante) et de la phase I (à allure constante), et

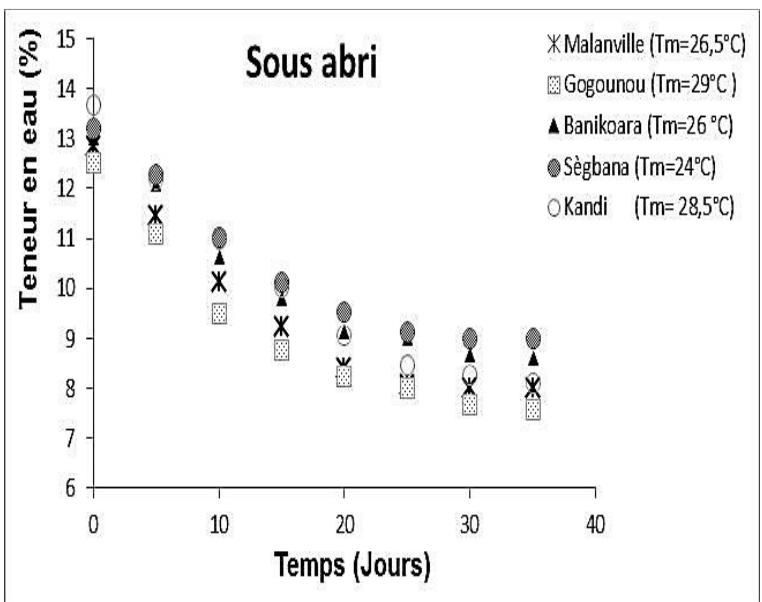

Figure 4 : Variation de la teneur en eau réduite en fonction du temns en nlein air

C'est un mécanisme complexe impliquant l'eau dans deux états liquide et vapeur, qui est souvent caractérisé par la diffusion effective. Cette propriété dépend de la température, de la pression et de la teneur en eau du produit (Moyne et al.,1992 ; Boudhrioua, 2004). Ces courbes montrent que la température a une influence sur l'évolution de la teneur en eau au cours du séchage. Une augmentation de la température entraine une diminution de la teneur en eau du produit. Ces échanges sont de moins en moins importants au fur et à mesure que le produit perd de l'eau et que le gradient d'humidité s'équilibre entre le produit et l'air ambiant. La différence la présence unique de la phase II (à allure décroissante) dans les courbes de séchage en plein air et sous abri du sorgho. Les mêmes résultats ont été obtenus pour différents produits végétaux (Belghit et al., 2000 ; Benhamou et al., 2008 ; Kouhila, 2001). Le séchage durant la phase à allure décroissante est régi par la diffusion d'eau dans le solide (Karel, 1997 ; Benhamou et al., 2008).

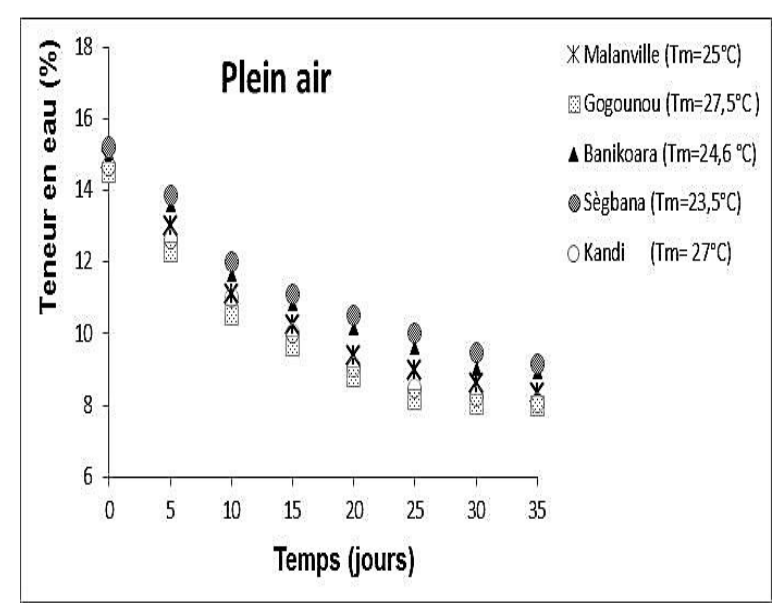

Figure 5 : Variation de la teneur en eau réduite en fonction du temns sous abri

de teneur en eau entre le produit et l'air est de plus en plus faible (Meziane, 2013). De même sur les figures 4 et 5 , on remarque que la diminution de la teneur en eau du produit sous abri est plus remarquable que celle observée en plein air. Cela est probablement dû à une parfaite diffusion de l'air à travers le produit hors sol, à la faible humidité relative de l'air et à la température de l'ambiance de séchage. Les figures 6 et 7 qui suivent présentent l'évolution de l'humidité relative de l'air asséchant et de la température de l'ambiance de séchage au cours du temps respectivement en plein air et sous abri. 


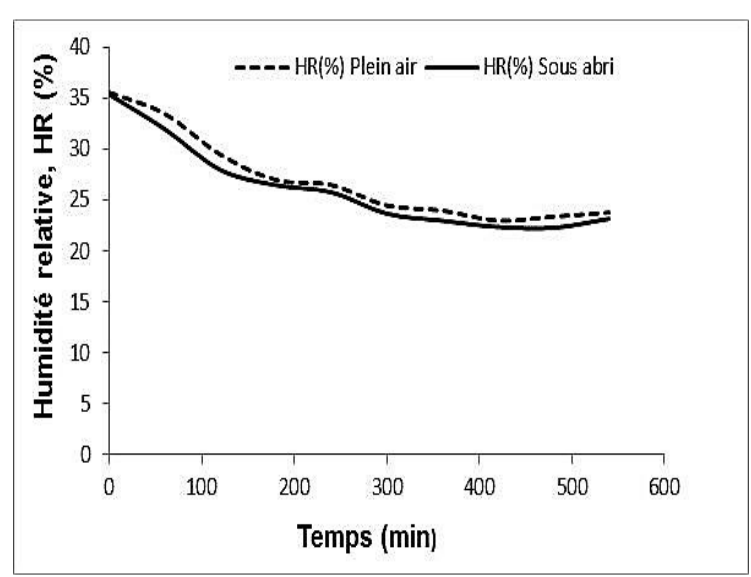

Figure 6 : Variation de l'humidité relative de l'air asséchant au cours du temps

Ces courbes montrent que l'écart entre l'humidité relative de l'air et la température de l'ambiance de séchage en plein air et sous abri est remarquable. L'humidité relative (HR) de l'air asséchant observée sous abri est plus faible que celle constatée en plein air. Nous remarquons également que l'évolution de l'humidité relative de l'air varie d'une façon opposée à celle de la température de l'air. En effet, les faibles valeurs de l'humidité relative de l'air constatées sous abri, constituent un facteur essentiel pour la durée de séchage et la qualité du produit. La température et l'activité de l'eau sont reconnues comme les principaux facteurs intervenant sur la stabilité au stockage et la durée de conservation des matières alimentaires.

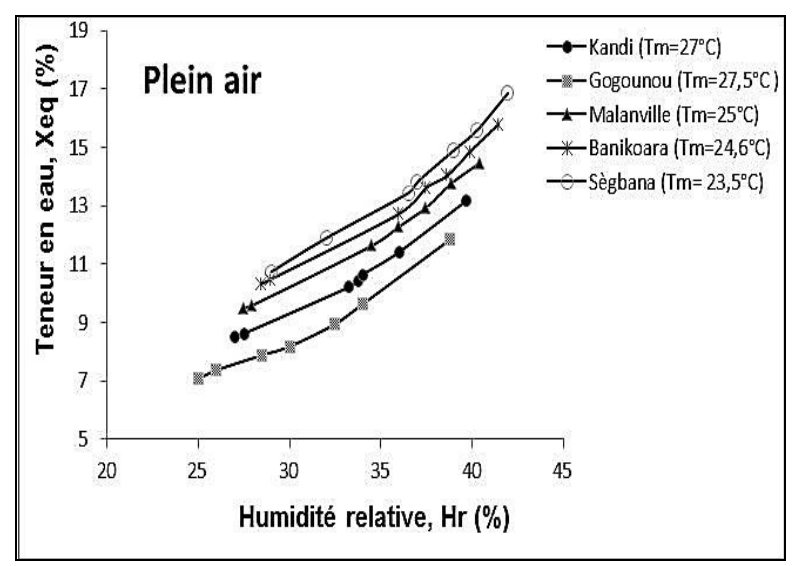

Figure 8 : Évolution de la teneur en eau en fonction de l'humidité relative de l'air asséchant en plein air

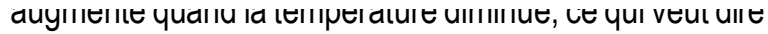
que le produit devient moins hygroscopique. Cela peut s'expliquer par l'augmentation de l'agitation thermique. En

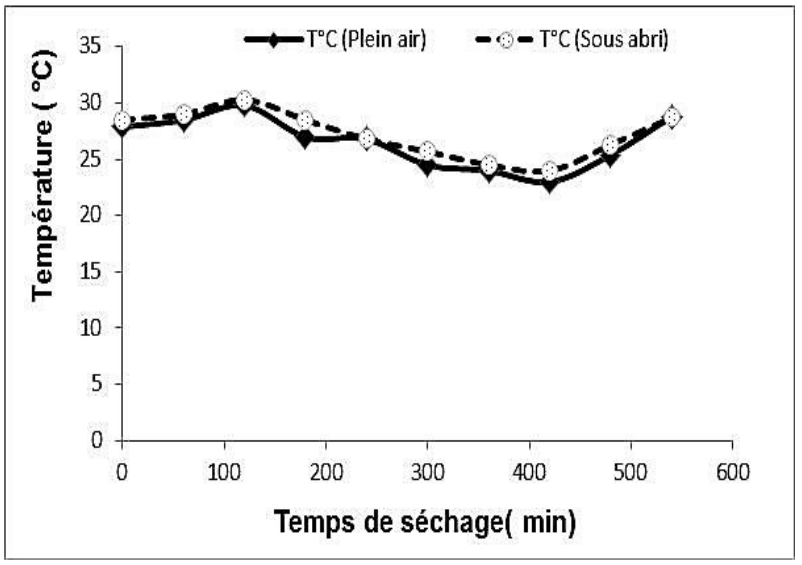

Figure 7 : Évolution de la température ambiante au cours du temps

Influence de l'humidité relative de l'air asséchant sur la teneur en eau du produit: La connaissance de l'activité de l'eau est d'un intérêt considérable en technologie alimentaire. La relation entre l'activité de l'eau et la teneur en eau, à température constante, est décrite pratiquement et théoriquement par l'isotherme de sorption de la vapeur d'eau (Ferradji et al., 2008). Les isothermes de sorption sont d'une grande importance dans les industries agro- alimentaires et notamment dans les opérations de séchage, de stockage et de conservation des aliments (Benhamou et al., 2010). Les figures 8 et 9 présentent les relations entre les humidités relatives de l'air asséchant et les teneurs en eau du produit respectivement en plein air et sous abri.

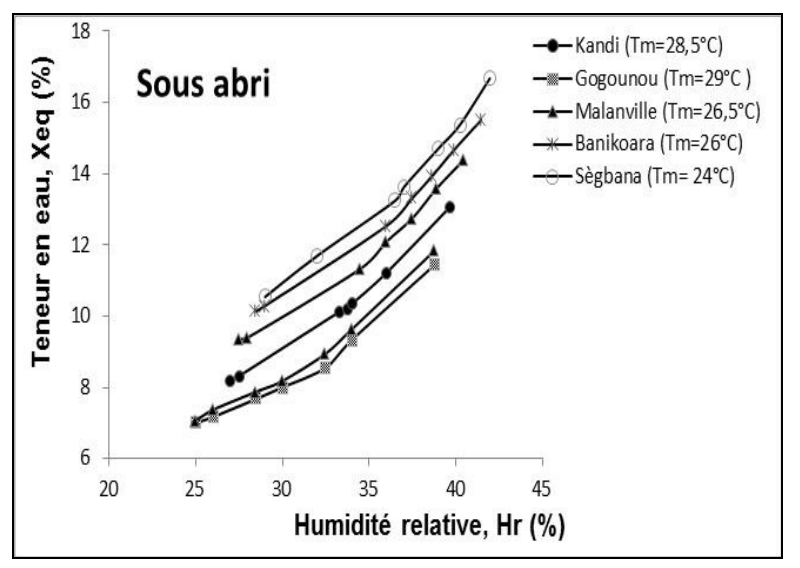

effet, pour des températures élevées, l'état d'excitation des molécules d'eau est plus important, entrainant une diminution des forces d'attraction des molécules d'eau entre elles (Lahsasni et al., 2003 ; Benhamou et al., 2010 ; Shittu et al., 2015). Cette observation pourrait être 

thermophysique et qualité microbiologique du produit séché

expliquée également par l'augmentation de la chaleur de sorption (énergie élevée de liaison de l'eau).

Caractérisation microbiologique du sorgho: Les analyses microbiologiques des échantillons du sorgho, juste à la post- récolte et séchés en plein air et sous abri sont respectivement mentionnées dans les tableaux 1, 2 et 3 . Les tableaux 2 et 3 révèlent une prédominance des germes aérobies mésophiles dans les échantillons de sorgho juste à la poste récolte, dans les localités de Kandi (47. 103 UFC/g), Gogounou (45. $10^{3} \mathrm{UFC} / \mathrm{g}$ ), et Malanville (37. $\left.10^{3} \mathrm{UFC} / \mathrm{g}\right)$. Une absence de ces germes dans les échantillons de Ségbana et de Banikoara a été observée. Les taux de levures et moisissures dénombrées dans ces mêmes échantillons sont inférieurs à ceux fixés par la norme AFNOR à l'exception de ceux de Malanville dans lequel les levures ont été dénombrées à $48 \mathrm{UFC} / \mathrm{g}$. Pour ces mêmes germes, nous constatons que le séchage en plein air a favorisé la prolifération de la flore totale qui passe de $37 \cdot 10^{3}$ à $43 \cdot 10^{3} \mathrm{UFC} / \mathrm{g}$ à Malanville et de $45.10^{3}$ à $65.10^{3} \mathrm{UFC} / g$ à Gogounou. Les levures passent de 48 à $92 \mathrm{UFC/g}$ à Malanville. Les levures sont absentes dans les échantillons de Gogounou avant le séchage ; on compte $23 \mathrm{UFC/g}$ en plein air. A Gogounou, on a assisté à une prolifération des moisissures au cours du séchage (41 UFC/g). Comparativement au séchage en plein air, le séchage sous abri a réduit le niveau de contamination des échantillons de sorgho en flore totale, en levures et en moisissures. En effet, Les germes fécaux absents avant le séchage, sont présents dans les échantillons en plein air. Ce constat pourrait s'expliquer par les conditions artisanales de stockage du sorgho. Sous abri les écarts sont nets avec une absence totale des germes fécaux dans les échantillons de sorgho. Staphylococcus aureus absent avant le séchage n'a pu apparaitre ni en plein air ni sous abri. Ces analyses microbiologiques effectuées sur différents échantillons de sorgho séchés ont permis d'établir que sa flore naturelle est constituée des bactéries mésophiles, des formes thermotolérantes. Pour les levures et moisissures, en dehors des Aspergilli, ces microorganismes font partie de ceux qui se retrouvent sur la plupart des produits alimentaires et ne présentent pas de risque de santé publique. Les levures et moisissures présentes font partie de la flore des céréales séchées. Les contaminateurs du sorgho peuvent provenir de la flore naturelle des téguments des céréales, des contacts avec le sol et le matériel de récolte, des animaux domestiques, de la poussière, et des manipulations consécutives. Olasupo et al. (1999) avaient préalablement noté dans leur étude sur le Wara (fromage traditionnel fabriqué au Nigéria) que le mode de production, de transformation et de manipulation pourrait impacter sur la qualité du produit. La présence des moisissures dans le produit est inhérente à la qualité de la matière première (le sorgho), et à la salubrité liée à la chaîne de transformation ou de conditionnement (OtengGyank, 1984).

Tableau 1 : Caractéristiques microbiologiques des échantillons de sorgho juste à la post- récolte

\begin{tabular}{l|c|c|c|c|c|c}
\hline \multicolumn{1}{c|}{ Germes } & Malanville & Gogounou & Kandi & Ségbana & Banikoara & $\begin{array}{c}\text { Norme AFNOR } \\
1996\end{array}$ \\
\hline Flore totale (UFC/g) & $37.10^{3}$ & $45.10^{3}$ & $47.10^{3}$ & $<1$ & $<1$ & $2.10^{2}$ \\
\hline $\begin{array}{l}\text { Coliformes totaux } \\
\text { (UFC/g) }\end{array}$ & $<1$ & 2 & 9 & $<1$ & $<1$ & 10 \\
\hline $\begin{array}{l}\text { Coliformes fécaux } \\
\text { (UFC/g) }\end{array}$ & $<1$ & $<1$ & $<1$ & $<1$ & $<1$ & $<1$ \\
\hline Levures (UFC/g) & 48 & $<1$ & $<1$ & 8 & 9 & 20 \\
\hline $\begin{array}{l}\text { Moisissures } \\
\text { (UFC/g) }\end{array}$ & $<1$ & $<1$ & $<1$ & $<1$ & $<1$ & 20 \\
\hline $\begin{array}{l}\text { Staphylococcus } \\
\text { aureus } \\
\text { (UFC/g) }\end{array}$ & $<1$ & $<1$ & $<1$ & $<1$ & $<1$ & $<1$ \\
\hline
\end{tabular}

Tableau 2: Caractéristiques microbiologiques des échantillons du sorgho après séchage au sol en plein air

\begin{tabular}{l|c|c|c|c|c|c}
\hline Germes & Malanville & Gogounou & Kandi & Ségbana & Banikoara & Norme AFNOR \\
\hline
\end{tabular}


Gandaho et al., J. Appl. Biosci. 2017 Séchage du sorgho produit dans le Nord-Bénin : Caractérisation thermophysique et qualité microbiologique du produit séché

\begin{tabular}{l|c|c|c|c|c|c}
\hline & & & & & & 1996 \\
\hline Flore totale & $43.10^{3}$ & $65.10^{3}$ & 300 & $<1$ & $<1$ & $2.10^{2}$ \\
\hline Coliformes totaux & $<1$ & 5 & 21 & $<1$ & $<1$ & 10 \\
\hline Coliformes fécaux & 3 & 8 & $<1$ & 15 & 32 & $<1$ \\
\hline Levures & 92 & 23 & $<1$ & - & $<$ & 20 \\
\hline Moisissures & $<1$ & 41 & $<1$ & $<1$ & $<1$ & 20 \\
\hline Staphylococcus aureus & $<1$ & $<1$ & $<1$ & $<1$ & $<1$ & $<1$ \\
\hline
\end{tabular}

Tableau 3 : Caractéristiques microbiologiques des échantillons du sorgho après séchage hors sol sous abri

\begin{tabular}{l|c|c|c|c|c|c}
\hline Germes & Malanville & Gogounou & Kandi & Ségbana & Banikoara & $\begin{array}{c}\text { Norme } \\
\text { AFNOR 1996 }\end{array}$ \\
\hline Flore totale & $37.10^{3}$ & $45.10^{3}$ & 84 & $<1$ & $<1$ & $2.10^{2}$ \\
\hline Coliformes totaux & $<1$ & $<1$ & $<1$ & $<1$ & $<1$ & 10 \\
\hline Coliformes fécaux & $<1$ & $<1$ & $<1$ & $<1$ & $<1$ & $<1$ \\
\hline Levures & 19 & $<1$ & $<1$ & $<1$ & $<1$ & 20 \\
\hline Moisissures & $<1$ & $<1$ & $<1$ & $<1$ & $<1$ & 20 \\
\hline Staphylococcus aureus & $<1$ & $<1$ & $<1$ & $<1$ & $<1$ & $<1$ \\
\hline
\end{tabular}

\section{CONCLUSION}

Les grains de sorgho séchés au soleil contiennent une flore microbienne naturelle variée, constituée des bactéries mésophiles, des formes thermotolérantes, des levures et moisissures. Le sorgho séché en milieu paysan sous abri semble présenter un caractère sanitaire acceptable mieux que celui séché à l'air libre. En plein air, l'humidité relative est plus élevée, devant les conditions environnementales peu reluisantes au sol (fréquentation des enfants et des animaux domestiques) et les contaminants imposent davantage leur loi. L'absence marquée des micro-organismes indicateurs de contamination sous abri laisse penser que l'hygiène $d u$ séchage est acceptable. L'absence des germes pathogènes (Staphylococcus aureus) et de la flore indicatrice de pollution fécale (Coliformes fécaux) par séchage sous abri confère au produit une qualité microbiologique acceptable. Toutefois, la présence de moisissure n'est pas favorable à la conservation du produit pour une longue durée. En effet, il s'agit d'un séchage naturel avec les aléas et les risques de contamination par la flore de l'environnement. Cependant, le sorgho séché se conserve relativement bien à température ambiante sous abri. Ces observations faites montrent qu'il existe une corrélation positive entre la teneur en eau du produit et la charge microbienne qu'il véhicule. La conservation du sorgho par le séchage en milieu paysan sous abri mérite d'être encouragée. Les tendances observées au niveau de la charge microbienne méritent d'être investiguées par des études ultérieures.

\section{REMERCIEMENTS}

Nous remercions les producteurs pour leur disponibilité et leur engagement à fournir des connaissances sur les techniques de stockage et de conservation des céréales.

\section{REFERENCES}

Ahouannou C, Jannot Y, Sanya E, Degan G, 2010. Détermination expérimentale et modélisation des isothermes de désorption de produits agricoles tropicaux. Afrique Science. 06 (3) : 117.

Belghit A, Kouhila M, Boutaleb BC, 2000. Experimental Study of Drying Kinetics by Forced Convection of Aromatic Plants. Energy Conversion and Management. 41(12) : 1303-1321.

Benhamou A, Idlimam A, Lamharrar A, Benyoucef B, Kouhila M, 2008. Diffusivité hydrique et cinétique de séchage solaire en convection forcée des feuilles de marjolaine. Revue des Énergies Renouvelables. 11 (1) : 75-85. 
Benhamou A, Kouhila M, Zeghmati B, Benyoucef B, 2010. Modélisation des isothermes de sorption des feuilles de marjolaine. Revue des Énergies Renouvelables. 13 (2) : 233-247.

Boudhrioua N., 2004. Étude des migrations de l'eau et du chlorure de sodium dans des gels alimentaires et influence de la composition du gel et du procédé appliqué. Thèse de Doctorat à l'École Nationale Supérieure des Industries Agricoles et Alimentaires. Massy, France. p 1-149.

Fandohan P, Hell K, Marasas WFO, Wingfield MJ, 2003. Infection of maize by Fusarium species and contamination with fumonisin. In africa Review African Journal of Biotechnology. 2 (12) : 570579.

Ferradji A, Acheheb H, Malek A, Hadjad N, 2008. Isothermes d'adsorption à $25^{\circ} \mathrm{C}$ et $45^{\circ} \mathrm{C}$ des pommes chargées de solutés et séchées. Revue des Énergies Renouvelables.11 (4) : 1-12.

Guiraud J et Galzy P, 1980. L'analyse microbiologique dans les industries alimentaires. Ed de l'usine : $183 \mathrm{p}$.

Karel M, 1988. Role of water activity in Food Properties and Computer-Aided. Engineering of Food Processing Systems, R. P. Sing, and A. G Medina, eds, Kluwer. Academic Publishers, Dodrecht, Boston and London : 135-156.

Kouhila M., 2001. Étude expérimentale et théorique de cinétiques de séchage convectif partiellement solaire des plantes médicinales et aromatiques (Menthe, Verveine, Sauge et Eucalyptus) de la région de Marrakech. Thèse de Doctorat d'État. Université Cadi Ayyad, Marrakech : p 1-170.

Lahsasni S, Koulia M, Fliyou M, 2003. Moisture adsorption-desorption isotherms of prickly pear cladode (Opuntia ficus indica) at different temperatures. Energ. Conv and Manag. 44: 923936.

Meziane S, 2013. Modélisation de la cinétique séchage convectif du grignon d'olive. Revue des Énergies Renouvelables. 16 (2) : 379-387.

Moyne CN, Kechaou PJ, Do Amaral Sobral M, Roques A, Bizot H, 1992. Séchage et Mécanismes de Transport de l'Eau dans les Gels. Entropies. 28(167) : 9-17.

Olasupo NA, Schillinger U, Narbard A, Dodd H, 1999. Occurrence of nisin $Z$ production in lactococcus lactis BFE 1500 isolated from Wara, a traditional Nigerian cheese production. Int. J. Food Microbiol. 53 (2) : 141-152.
Oteng-Gyank K, 1984. Introduction à la microbiologie alimentaire dans les pays chauds. Collection technique et documentation. Lavoisier : Paris : 260p.

Shittu TA, Idowu-Adebayo F, Adedokun II, Alade O, 2015. Water vapor adsorption characteristics of starch albumen powder and rheological behavior of its paste. Nigerian Food Journal 33: 90-96.

Touzi A, Merzaia-Blama A, 2008. Conservation des denrées agroalimentaires par séchage dans les régions sahariennes. Revue des Énergies Renouvelables SMSTS'08 : 267-272.

Yiannikouris A, Jouany J-P, 2002. Les mycotoxines dans les aliments des ruminants, leur devenir et leurs effets chez l'animal. INRA Prod. Anim. 15 (1) : 3-16 